\title{
Evaluation of Preliminary Design Review (PDR) formats in student space projects.
}

\author{
E. Menting, T. Britting, L. Pepermans \\ Delft Aerospace Rocket Engineering (DARE) \\ The Netherlands \\ PRG@dare.tudelft.nl
}

In any engineering project, the Preliminary Design Review (PDR) is a major milestone. The design is presented to nonproject members who provide feedback. This feedback is used by the team to incorporate in the detailed design. The review can be done using written documentation and/or an oral presentation.

Student space projects often have a tight timeline which imposes constraints on the PDR process. Depending on this timeline and the availability of team members and reviewers, the PDR can be done in several ways. Variations can be made in: level of detail in documentation beforehand, the time between sending documentation and PDR, the format of the feedback and the implementation of the feedback.

The article originates from the widely varying PDR formats the authors experienced in different student space projects. Within Delft Aerospace Rocket Engineering (DARE) a PDR was held on both the Stratos III and Stratos IV student-built sounding rockets. Furthermore, a PDR on the Supersonic Parachute Experiment Aboard REXUS (SPEAR) mission was held both within DARE internally and within the REXUS/BEXUS program.

In the article, the pros and cons of the various formats are discussed together with their applications. As cases, the REXUS/BEXUS and DARE PDR formats are compared to each other and the ESCC standard.

Keywords- Preliminary Design Review, PDR, student project, DARE, REXUS, ECSS.

\section{INTRODUCTION}

During the Supersonic Parachute Experiment Aboard REXUS (SPEAR) project, the team experienced a different format of the Preliminary design review (PDR) in the REXUS/BEXUS program than it was used to within DARE. The different setups also resulted in different feedback. This led the team to wonder if these formats can be altered and combined in a way to tailor to a student project's needs. This paper explains key aspects of both reviews and evaluates some of their pros and cons.

The process of a review in space projects is also streamlined by the ECSS guidelines. The paper will provide a short summary of the process presented in ECSS and what the advantages of this method can be for student space projects.

\section{DARE}

DARE is a student rocketry society with approximately 150 members. These members work in multiple teams and projects. R\&D teams have around 10-15 members whilst flagship projects like Stratos have around 60 members, distributed over different technical and non-technical sub teams.

The design cycles in DARE consist of a conceptual, preliminary and detailed design. The conceptual design phase is mostly only reviewed within the team itself, where during the PDR all members of DARE and, on occasion, external reviewers from the Technical University of Delft or companies are invited.

The design is shown during a presentation in which the design trade-offs and choices are explained. After each section, a question and discussion round is started in which the reviewers can question and explore the design. The reviewers' experience with similar (sub-)systems leads to

recommendations for the design which is one of the key points during this review process.

Main characteristics of a DARE PDR are:

- No or limited documentation is sent beforehand.

- If documentation is sent, this is only done shortly before the PDR (one to two days).

- Low number of external reviewers is present.

- The presentation on the design and design choices is relatively long and can last up to two hours for a subsystem.

- The feedback from the reviewers is not structured in categories.

- There is little to no time for the reviewers to let the design sink in and generate questions or comments.

- There is much freedom to dive into different discussions and reviewers can bring up new ideas or solutions to the problems of the team. There is room to discuss this elaborately.

- Reviewers usually have many questions on the design after the presentation, as it does not fully cover all the details.

- The level of detail of the design in a PDR is not predetermined, thus varies often. Sometimes the design is still more in the conceptual design phase. 
The DARE PDR's as they were, were long and somewhat ineffective. As the reviewers did not have proper documentation or time to read the provided documentation, questions were often not relevant or very broad. This led to the presenter spending the majority of the PDR time on explaining the mission and the conceptual design and less time on the design issues. This was made worse by the observation that many members had different opinions on what the preliminary design needed to contain.

Based upon the REXUS selection workshop and the REXUS PDR, a first improvement to the DARE PDRs were made. Changes included:

- Documentation is sent out at least one week beforehand.

- The PDR starts with an overview of the mission.

- The PDR contains a 30-minute presentation on the final preliminary design broken up in clear subsystems.

- Discussions and questions are done per subsystem.

- More in-depth content discussion than usual DARE reviews were possible.

- Reviewers can give written feedback on documentation if they aren't able to come to the PDR.

The modification of the DARE PDR format led to a better structure and noticeably better results for both the SPEAR internal PDR.

\section{REXUS}

Within the REXUS/BEXUS program Cycle 12, the SPEAR team had its PDR in February 2019. Within the REXUS program, the student team hands in an updated version of their Student Experiment Documentation (SED) two weeks before the review. The SED contains the full preliminary design and progress up to that point. The PDR is held with the student team and a panel from the REXUS/BEXUS program containing multiple members of the different organising parties and different specialties. During the PDR, the student team gives a short presentation of 20 minutes to summarise the design and any design changes that were made between the SED and the PDR. After this, the panel gives their feedback on the design and the documentation in an hour during which the team makes notes. This means the review is relatively one-sided, in the presentation from the team towards the panel and vice versa for the feedback. Minutes of the feedback, including feedback on the SED, is sent to the team a few weeks after the design review.

In the same week as the PDR, a Q\&A session with all student teams of that cycle and the REXUS panel is organised. Here issues with the design or controversial points can be discussed in more detail where also alternative solutions can be introduced.

\section{Main characteristics of the REXUS PDR:}

- The documentation (SED) is provided two weeks prior to the review.

- High paced student project means content can actually change significantly in those two weeks.
- PDR itself is somewhat one-sided.

- Review and comments are more in-depth as elaborate documentation is read and studied beforehand.

- Q\&A session with the panel allows for more in-depth discussions and is a much-needed supplement to the PDR.

- Elaborate minutes, including action points, are sent after the review, enabling the team to ensure the feedback was interpreted correctly.

- The content of the feedback on the written documentation is different compared to DARE's. It goes into the design much deeper and also focuses on details, not solely on the basic working principle. Aside this it also includes feedback on the quality of documentation itself.

\section{ECSS}

The European Cooperation for Space Standardization created an extensive set of guidelines meant to improve consistency and facilitate design efforts within the European space sector. The published standards on design reviews are discussed in the following section, which is based on ECSS-M-ST-10-01C 'Organization and conduct of reviews' [1].

The overall purpose of a review is to examine or evaluate the current status of the project in which the problems faced throughout the design process are discussed. Reviews often act as a moment of reflection.

The ECSS identifies three different steps during the review process:

- 'Review initiation'

- 'Review data package preparation and distribution' allows the review participants, REXUS, to familiarize with the to be reviewed documents.

- 'Review of the documentation' includes a kick-off presentation during which RID-forms (Review Item Discrepancy) are used. The issues, remarks and questions from the RID-forms are afterwards addressed during meetings between both parties. The review team, SPEAR, is then expected to compile all results and conclusions that arose from the review.

- 'Review findings and conclusion' covers the examination whether the objectives of the review were met.

ECSS-M-ST-10-01C includes a list of requirements that serve as guidelines to a productive review for both the review team and review participants. The general requirements serve mainly to make sure that constructive feedback is exchanged and that any issues are correctly addressed.

The four following roles should be filled during a review:

- The review authority approves the review procedures, examines review reports, makes the recommendations 
and decides whether or not the review objectives have been met.

- The customer ensures that the review can be held by providing the necessary means, such as a data management system for the review data.

- The supplier's role is to provide all logistics, documentation, data and RIDs for the review.

- The review team leader mainly manages the review teams' activities and approves the RIDs.

- The review team reviews the documents and subsequently provides feedback using the RID-forms.

The chronological order of a review should be as following:

- A prerequisite key point serves as a check that the review can start.

- The kick-off meeting introduced the to be reviewed documents and product

- During the coordination meeting, the documents are reviewed, and all RID-forms are released.

- In a collocation meeting, the RIDs are discussed, following with action points that should be undertaken.

- After the review team close-out meeting, the results from the previous meeting should be summarized

- Finally, the review authority meeting confirms the outcome of the review and creates a review authority report.

The final steps of a review include the follow-up of action points and processing of the feedback received through the RID-forms.

\section{COMPARISON OF PDR FORMATS}

When comparing the three PDR styles; Old DARE, New DARE, and REXUS, one can see that where the Old DARE style allowed for too much discussion, the REXUS format however does not allow for interaction during the PDR. The latter is solved by the introduction of a Q\&A session during the REXUS training week. This however, is not feasible in most student projects as there are often insufficient external specialists.

For most student space projects, it is unfeasible to follow the cycle proposed by ECSS as this takes place in a much longer time span. A much larger part of the ECSS process takes place on paper, with meetings to coordinate the process. In a student project it is still recommended that the one PDR moment stays central in the review process. However, some advantages are identified, of which the RID-forms is the most significant one, allowing for structured written feedback.

Both the REXUS and the ECSS formats include documentation to be sent out before the PDR. However, in the REXUS PDR format this means that the team should not change the design or documentation for this period. Given the short time span of a student space project, two weeks is quite long in a high paced student project. The use of RID-forms creates a compromise where the documentation is sent out 2 weeks before the PDR, but the team can still updates the design in this period.

\section{RECOMMENDATIONS}

For student space projects, the team proposes the following set up that is efficient and thorough but still allows for implementation into student projects.

- $\mathrm{T}-3 \mathrm{w}-$ Reviewers are identified and asked. Important is to ensure a broad scope and experience of the review board.

- $\mathrm{T}-2 \mathrm{w}-$ Documentation is sent to reviewers

- T-1w - Final call for RID forms

- T-0 - Presented PDR

- $\mathrm{T}+1 \mathrm{w}-$ Send out minutes of PDR

- $\mathrm{T}+2 \mathrm{w}-$ Final feedback on minutes

- $\mathrm{R}+3 \mathrm{w}-\mathrm{PDR}$ phase finished

The PDR itself should be set up such that the following topics are discussed:

- Overview of mission and concepts to ensure all reviewers are on the same page

- Overview of the RID forms that are not solved or addressed with explanation

- Preliminary design overview of the system and subsystems

- Preliminary design of the system interfaces

- Overview of risks

- Overview of budget

- Question round organised per topic

Even though this set up requires a total of six weeks, it provides the team with two feedback moments. As the first one is written, it allows for the team to tackle these before the presented PDR. When the points mentioned above are followed the presented PDR gives a complete and total overview of the project. By adding an introduction talk on the mission and the conceptual design, the team can ensure the review board is on the same page and that a more efficient PDR can be held. The inclusion of the RID-forms allows the team to start addressing issues in the two weeks prior to the presented PDR. This removes the downside identified in the REXUS PDR style.

By sending the minutes to the reviewers, the team can ensure that the written down feedback is as the review board intended it.

Most importantly, the Preliminary Design Review should be planned as a review phase, not a review moment. This setup also allows for a good processing of the received feedback instead of having to directly continue with the next design phase. 


\section{CONCLUSIONS}

Student teams should never underestimate the usefulness of design reviews during their space project if done well. This is maybe even more important for the PDR than for any other review. Given the three different styles of design reviews, the team proposes a combination of the ECSS and the REXUS review. This allows for more discussion during the PDR, alike the DARE model.

\section{REFERENCES}

[1] European Space Agency for the members of ECSS. (2008) ECSS-M-ST10-01C Organisation and conduct of review, retrieved from https://ecss.nl/. 